NBER WORKING PAPER SERIES

AGRO-ECOSYSTEM PRODUCTIVITY AND THE DYNAMIC RESPONSE TO SHOCKS

\author{
Jean-Paul Chavas \\ Working Paper 22624 \\ http://www.nber.org/papers/w22624 \\ NATIONAL BUREAU OF ECONOMIC RESEARCH \\ 1050 Massachusetts Avenue \\ Cambridge, MA 02138 \\ September 2016
}

This paper was supported in part by a grant from the Graduate School, University of Wisconsin, Madison. The views expressed herein are those of the author and do not necessarily reflect the views of the National Bureau of Economic Research.

NBER working papers are circulated for discussion and comment purposes. They have not been peer-reviewed or been subject to the review by the NBER Board of Directors that accompanies official NBER publications.

(C) 2016 by Jean-Paul Chavas. All rights reserved. Short sections of text, not to exceed two paragraphs, may be quoted without explicit permission provided that full credit, including ()$^{\circ}$ notice, is given to the source. 
Agro-Ecosystem Productivity and the Dynamic Response to Shocks

Jean-Paul Chavas

NBER Working Paper No. 22624

September 2016

JEL No. O13,O3,Q1

\begin{abstract}
$\underline{\text { ABSTRACT }}$
This paper investigates the nonlinear dynamic response to shocks, relying on a threshold quantile autoregression (TQAR) model as a flexible representation of stochastic dynamics. The TQAR model can identify zones of stability/instability and characterize resilience and traps. Resilience means high odds of escaping from undesirable zones of instability toward zones that are more desirable and stable. Traps mean low odds of escaping from zones that are both undesirable and stable. The approach is illustrated in an application to the dynamics of productivity applied to historical data on wheat yield in Kansas over the period 1885-2012. The dynamics of this agroecosystem and its response to shocks are of interest as Kansas agriculture faced major droughts, including the catastrophic Dust Bowl of the 1930's. The analysis identifies a zone of instability in the presence of successive adverse shocks. It also finds evidence of resilience. We associate the resilience with induced innovations in management and policy in response to adverse shocks.
\end{abstract}

Jean-Paul Chavas

University of Wisconsin

Department of Agriculture \& Applied Economics

Taylor Hall, 427 Lorch Street

Madison, WI 53706

jchavas@wisc.edu 


\section{Agro-Ecosystem Productivity and the Dynamic Response to Shocks}

\section{Introduction}

Dynamics is at the heart of economic development and the search for processes that contribute to improving human welfare. But dynamic process are typically complex, especially under nonlinear dynamics. Indeed, nonlinear dynamic systems can exhibit many patterns. For deterministic systems, this can go from reaching a unique steady state, to having multiple steady states, to displaying limit cycles, or even to being chaotic (e.g., May, 1976). For stochastic systems, the complexity increases further, making it challenging to evaluate the dynamic response to unanticipated shocks. The assessment of such dynamic response is highly relevant in economics. Some shocks are favorable (e.g., good weather, the discovery of new knowledge) with positive impacts on welfare both in the short run and the longer run. But other shocks have a negative impact on human welfare (e.g., drought, disease). The dynamic effects of such shocks has been of great interest to economists and policy makers. Under some scenarios, their adverse effects matter in the short run but dissipate in the longer run. But under other scenarios, their longer term impacts can be sustained and large. An example is the case of poverty traps which associate poverty with reduced investment and meager prospects for economic growth (e.g., Barrett and Carter, 2013; Kraay and McKenzie, 2014). Another example is from ecology: under some circumstances, an ecosystem may fail to recover under extreme shocks (e.g., Holling, 1973; Common. and Perrings, 1992; Perrings, 1998; Gunderson, 2000; Folke et al., 2004; Derissen et al., 2011). Other examples include cases of economic collapse with large and lasting adverse effects on society (e.g., Tainter, 1990; Diamond, 2005). In these examples, the outcomes are all undesirable. But the assessment of these situations can be challenging for two reasons: 1/ 
such adverse scenarios may not be very common; and 2/ the dynamics of the underlying process is often complex and poorly understood. This suggests two useful directions of inquiry. First, we need to refine our tools used in dynamic analysis. Second, we need to explore applications that may provide new insights into economic dynamics. These two directions are key motivations for this paper.

This paper studies nonlinear dynamics in economics. It makes three contributions. First, it explores new ways to analyze stochastic dynamics. The focus is on representations of stochastic dynamics that allow for flexible dynamic response to shocks. The analysis relies on a threshold quantile autoregression (TQAR) model (Galvao et al., 2011; Chavas and Di Falco, 2016). The TQAR model is empirically tractable. And it is flexible: it allows dynamics to vary with both current shocks and past states. As such, a TQAR model can be used to assess how dynamics can differ across situations (as reflected by different shocks and different states). This makes it particularly appropriate for our purpose.

A second contribution is to use our proposed approach to identify zones of stability/instability and to characterize resilience and traps. Resilience means good odds of escaping from undesirable zones of instability toward zones that are more desirable and stable. Traps mean low odds of escaping from zones that are both undesirable and stable. As such, resilience is desirable, but traps are not. We argue that the TQAR model provides a good basis to evaluate the resilience of a system and the presence of traps.

A third contribution is to illustrate the usefulness of our approach in an application to the dynamics of an agro-ecosystem. Our empirical analysis uses historical data on wheat yield in Kansas during the period 1885-2012. Historically, the Western Great Plains have experienced many periods of severe drought (Burnette and Stahle, 2013). The worse drought occurred in the 
1930's leading to the Dust Bowl, a major American environmental catastrophe (Hornbeck, 2012). Coupled with intensive land use, the drought led to major crop failure, wind erosion and dust storms. The impact was particularly severe in Kansas, where land erosion contributed to significant decrease in land value and agricultural productivity (Hornbeck, 2012). The short run response to the environmental destruction was mostly population migration away from the affected areas. But the long run effects were major and lasting. Hornbeck (2012) documents that soil erosion due to the Dust Bowl contributed to a decline in land value up to 30 percent in the long term. Wheat being the major crop in Kansas (USDA, 2015), studying Kansas wheat yield provides a great case study of the dynamic response to environmental shocks. Of special interest are the effects of extreme shocks both in the short run and in the long run. In the context of wheat yield, our analysis identifies a zone of instability in the presence of successive adverse shocks. It also finds evidence of resilience. We associate the resilience with induced innovations in management and policy in response to adverse shocks. This indicates the importance of management and policy in the dynamic response to shocks.

\section{Dynamics}

Consider a dynamic system evolving according to the state equations

$$
\begin{aligned}
& y_{t}=h\left(y_{t-1}, \ldots, y_{t-p}, z_{t}\right), \\
& z_{t}=g\left(y_{t-1}, \ldots, y_{t-p} ; z_{t-1}, \ldots, z_{t-p}\right),
\end{aligned}
$$

where $y_{t} \in \mathbb{R}$ measures payoff at time $t, z_{t}$ is a vector of variables affecting the system with dynamics given in (1b), and $p \geq 1$. Equations (1a)-(1b) provide a general representation of dynamics, allowing for joint dynamics in payoff $y_{t}$ and in the state variables $z_{t}$. After successive substitutions of (1b), note that equation (1a) can be alternatively written as 


$$
\begin{aligned}
y_{t} & =h\left(y_{t-1}, y_{t-2}, \ldots, g\left(y_{t-1}, y_{t-2}, \ldots ; z_{t-1}, z_{t-2}, \ldots\right)\right) \\
& =h\left(y_{t-1}, y_{t-2}, \ldots, g\left(y_{t-1}, y_{t-2}, \ldots ; g\left(y_{t-2}, \ldots ; z_{t-2}, \ldots\right), z_{t-2}, \ldots\right)\right) \\
& =\cdots \\
& =f_{0}\left(y_{t-1}, y_{t-2}, \ldots ; y_{0}, z_{0}\right)
\end{aligned}
$$

where $\left(y_{0}, z_{0}\right)$ are initial conditions which we take as given. Assume that the effects of lagged values of $y_{t-j}$ on $y_{t}$ in (2) become negligible for all $j>m$. It follows that equation (2) can be written as

$$
y_{t}=f\left(y_{t-1}, \ldots, y_{t-m}, e_{t}\right)
$$

and $e_{t}$ is a random variable representing unobservable effects at time $t$. We assume that $e_{t}$ is identically and independently distributed ${ }^{1}$ with a given distribution function.

Equation (3) is a $m$-th order stochastic difference equation representing economic dynamics under general conditions. Comparing equations (1) and (3), equations (1a)-(1b) are structural equations describing how the system evolves over time, while equation (3) is a reduced form equation of the same system. While equation (3) does not reflect structural information about the system, it has two advantages: 1 / it provides a valid representation of the system dynamics; and 2/ it does not require information about the variables $z_{t}$. This is significant advantage when some of the dynamic factors affecting payoff are not observable. For this reason, our analysis will focus on the reduced form representation (3).

Note that, equation (3) can be written as the first-order difference equation

$$
w_{t} \equiv\left[\begin{array}{c}
y_{t} \\
\vdots \\
y_{t-m+1}
\end{array}\right]=\left[\begin{array}{c}
f\left(y_{t-1}, \ldots, y_{t-m}, e_{t}\right) \\
\vdots \\
y_{t-m+1}
\end{array}\right] \equiv H\left(w_{t-1}, e_{t}\right)
$$

where $w_{t} \in \mathbb{R}_{+}^{m}$. Equation (4) can be used to characterize the nature of dynamics. Under differentiability, let $D H\left(w_{t-1}, e_{t}\right)=\partial H\left(w_{t-1}, e_{t}\right) / \partial w_{t-1}$ be a $(m \times m)$ matrix. Denote the 
characteristic roots of $D H\left(w_{t-1}, e_{t}\right)$ by $\left[\lambda_{1}\left(w_{t-1}, e_{t}\right), \ldots, \lambda_{m}\left(w_{t-1}, e_{t}\right)\right]$ where $\left|\lambda_{1}\left(w_{t-1}, e_{t}\right)\right| \geq$ $\cdots \geq\left|\lambda_{m}\left(w_{t-1}, e_{t}\right)\right|,\left|\lambda_{j}\right|$ being the modulus of the $j$-th root, $j=1, \ldots, m$, and $\lambda_{1}$ being the dominant root.

Where equation (3) is linear in $\left(y_{t-1}, \ldots, y_{t-m}\right)$, the system exhibits linear dynamics. In this case, the matrix $D H$ is constant and so are its roots $\left(\lambda_{1}, \ldots, \lambda_{m}\right)$. Consider for a moment a situation where $e_{t}$ is constant for all $t$. Then, under linear dynamics, the system is globally stable (in the sense that $\lim _{t \rightarrow \infty} y_{t}=y^{e}$ for any initial condition $y_{0}$ ) if $\left|\lambda_{1}\right|<1$ (Hasselblatt and Katok, 2003). Alternatively, the system would be unstable if $\left|\lambda_{1}\right|>1$. When $\lambda_{1}$ is real, the dynamics of $y_{t}$ has a forward path that is $\left\{\begin{array}{c}\text { exponential } \\ \text { oscillatory }\end{array}\right\}$ when $\lambda_{1}\left\{\begin{array}{l}>0 \\ <0\end{array}\right\}$. And when $\lambda_{1}$ is complex, then $\lambda_{1}=$ $a+b \sqrt{-1}$ and the system exhibits cyclical dynamics, with a cycle of period $[2 \pi / \operatorname{arctg}(b / a)]$. In the general case where equation (3) is nonlinear in $\left(y_{t-1}, \ldots, y_{t-m}\right)$, the system exhibits nonlinear dynamics. Under nonlinear dynamics, the forward path of $y_{t}$ can exhibit a variety of dynamic patterns. For example, holding $e_{t}$ constant for all $t, y_{t}$ can eventually reach a unique steady state, it can have multiple steady states, it can exhibit limit cycles, or it can be chaotic (e.g., May, 1976). Situations of multiple steady-state equilibria have been of interest. Multiple steady-sates $\left(y_{1}^{e}, \ldots, y_{M}^{e}\right)$ would arise if $\lim _{t \rightarrow \infty} y_{t}=y_{j}^{e}$ when $y_{0} \in S_{j}, j=1, \ldots, M$, where $M>1$ and $\left(S_{1}, \ldots, S_{M}\right)$ is a partition of $\mathbb{R}$. In this context, the set $S_{j}$ is the attractor of point $y_{j}^{e}$, $j=1, \ldots, M$, as having initial condition $y_{0}$ in $S_{j}$ eventually leads to $y_{j}^{e}, j=1, \ldots, M$. When a steady-state $y_{j}^{e}$ is identified as being "undesirable", it means that it is good to avoid being in the set $S_{j}$. Examples include cases of ecological collapse in ecology (Holling, 1973) and poverty trap in economics (Barrett and Carter, 2013; Kraay and McKenzie, 2014). 
Under nonlinear dynamics, both $D H\left(w_{t-1}, e_{t}\right)$ and the dominant root $\lambda_{1}\left(w_{t-1}, e_{t}\right)$ depend on the evaluation point $\left(w_{t-1}, e_{t}\right)$. In general, $\ln \left(\left|\lambda_{1}\left(w_{t-1}, e_{t}\right)\right|\right)$ measures the rate of divergence in $y_{t}$ along forward paths in the neighborhood of $\left(w_{t-1}, e_{t}\right)$ (Hasselblatt and Katok, 2003). In this context, the dynamic properties just discussed still apply but only locally, i.e. in the neighborhood of $\left(w_{t-1}, e_{t}\right)$ : the dynamics is locally stable if the dominant root satisfies $\left|\lambda_{1}\left(w_{t-1}, e_{t}\right)\right|<1$; and it is locally unstable if $\left|\lambda_{1}\left(w_{t-1}, e_{t}\right)\right|>1$. We will make use of these local properties in our empirical analysis below.

The analysis of dynamics becomes more challenging in the stochastic case: the random vector $e_{t}$ in (3) affects the path of $y_{t}$ over time. This is relevant when $e_{t}$ represents unanticipated shocks. In this context, a key question is: What is the dynamic response of the system (3) to a shock $e_{t}$ ? This is the essence of the concept of resilience. A resilient system is defined as a system that can recover quickly from a shock (Holling, 1973;). This gains importance in the presence of adverse shocks (Di Falco and Chavas, 2008; Chavas and Di Falco, 2016). For example, in ecology, a resilient system would recover quickly from an adverse shock; but a nonresilient system may collapse. In economics, a resilient household would recover quickly from an adverse income shock; but a non-resilient household would not. While adverse shocks always have negative short term effects, resilience means such effects would eventually disappear in the longer term. But non-resilient systems would behave differently: they would see persistent longer term effects.

The dominant root $\lambda_{1}\left(w_{t-1}, e_{t}\right)$ provides useful insights on system dynamics. We discuss three cases. First, consider the case where $\lambda_{1}\left(w_{t-1}, e_{t}\right)$ is close to 0 for all $\left(w_{t-1}, e_{t}\right)$. This system would exhibit little dynamics and any shock would have minor or no long term effects. In a second case, assume that $\left|\lambda_{1}\left(w_{t-1}, e_{t}\right)\right|$ is positive but less than 1 for all $\left(w_{t-1}, e_{t}\right)$. Then, there 
would be a dynamic response to any shock. But having $\left|\lambda_{1}\left(w_{t-1}, e_{t}\right)\right|<1$ means that the impact of a shock would die down over time and eventually disappear in the long term. In this case, the magnitude of the dominant root remains useful. Having $\left|\lambda_{1}\left(w_{t-1}, e_{t}\right)\right|$ close to 0 (close to 1$)$ means a rapid (slow) decay of the temporal effects of a shock. In other words, a rise in $\left|\lambda_{1}\left(w_{t-1}, e_{t}\right)\right| \in(0,1)$ corresponds to stronger impacts of a shock in the intermediate term. Third, consider the case where $\left|\lambda_{1}\left(w_{t-1}, e_{t}\right)\right|$ is greater than 1 for some $\left(w_{t-1}, e_{t}\right)$. As discussed above, this corresponds to local instability in the neighborhood of $\left(w_{t-1}, e_{t}\right)$. A possible situation is that this local instability varies with the neighborhood. To illustrate, consider a system where $N_{1}, N_{2}, N_{3}$ are three different neighborhoods where $\left|\lambda_{1}\left(w_{t-1}, e_{t}\right)\right|<1$ when $\left(w_{t-1}, e_{t}\right) \in N_{1} \cup N_{3}$ but $\left|\lambda_{1}\left(w_{t-1}, e_{t}\right)\right|>1$ when $\left(w_{t-1}, e_{t}\right) \in N_{2}$. This system exhibits local stability in neighborhoods $N_{1}$ and $N_{3}$, but local instability in neighborhood $N_{2}$. Local instability in $N_{2}$ means that dynamics would tend to move $y_{t}$ away from $N_{2}$. In situations where $N_{2}$ is surrounded by $N_{1}$ and $N_{3}$, this would identify points in $N_{2}$ as tipping points, i.e. as points where $y_{t}$ would tend to escape from as they move toward locally stable neighborhoods. In this case, knowing which locally stable neighborhood $\left(N_{1}\right.$ or $\left.N_{3}\right)$ is more likely to be visited would be of interest. For example, if being in $N_{1}$ is seen as being undesirable, then an escape from $N_{2}$ to $N_{3}$ would be seen as a better scenario than moving from $N_{2}$ to $N_{1}$.

These patterns are illustrated in Figure 1 under four scenarios. Figure 1 shows how $\left|\lambda_{1}\right|$ can vary with $e_{t}$, where higher (lower) values of $e_{t}$ are interpreted as favorable (unfavorable) shocks. The first scenario is the case where $\lambda_{1}$ is constant. This occurs when the dynamics is represented by a linear autoregressive (AR) process, in which case the dynamic response to shocks does not depend on the situation considered Scenarios 2-4 are associated with nonlinear dynamics where $\lambda_{1}$ is not constant. Scenario 2 exhibits a pattern where $\left|\lambda_{1}\right|$ has an inverted U- 
shape with respect to $e_{t}$, with a zone of instability (where $\left|\lambda_{1}\right|>1$ ) surrounded by two zones of stability (where $\left|\lambda_{1}\right|<1$ ): a favorable zone (where $e_{t}$ is high), and an unfavorable zone (where $e_{t}$ is low). It means that the forward path of $y_{t}$ would tend to escape from the instable zone. And in the case where there is a low probability of escaping from the unfavorable stable zone, this would identify this zone as a trap. Scenario 3 shows a situation where there is a zone of instability but it occurs only for low values of $e_{t}$. This is an example of resilience where the dynamics would move the system away from unfavorable outcomes. Finally, Scenario 4 shows a situation where there is zone of instability but it occurs only for high values of $e_{t}$. This represents a collapse where the dynamics moves the system away from favorable outcomes. These examples illustrate that many patterns of dynamics are possible. ${ }^{2}$ Note that the dynamics would gain additional complexities when we note that $\left|\lambda_{1}\left(w_{t-1}, e_{t}\right)\right|$ can vary with both $e_{t}$ and $w_{t-1}$. The empirical challenge to evaluating these complexities is addressed in section 3 below.

Under stochastic dynamics, a related issue is: What are the implications of dynamics for the distribution of $y_{t}$ in the long run? To address this question, note that the dynamics in (3) can be alternatively written in terms of a Markov chain (Billingsley, 1961; Meyn and Tweedie, 1993). Consider partitioning the space $\mathbb{R}$ into $\mathrm{K}$ mutually exclusive intervals $\left\{v_{1}, \ldots, v_{K}\right\}$. To illustrate, consider the case where $m=1$. Letting $M=\{1, \ldots, K\}$, we have

$$
\begin{gathered}
\operatorname{Prob}\left(y_{t} \in v_{i}\right)=\sum_{j \in M}\left\{\operatorname{Prob}\left[y_{t} \in v_{i} \mid y_{t}=f\left(y_{t-1}, e_{t}\right), y_{t-1} \in v_{j}\right]\right. \\
\left.\operatorname{Prob}\left[y_{t-1} \in v_{j}\right]\right\}
\end{gathered}
$$

for $i \in M$. Under time invariance, equation (5a) can be written as the Markov chain model

$$
p_{t}=A p_{t-1}
$$


where $p_{t}=\left(p_{t, 1}, \ldots, p_{t, K}\right)^{\prime}$ is a $(K \times 1)$ vector with $p_{t, j}=\operatorname{Prob}\left(y_{t} \in v_{j}\right), j \in M$, and $A$ is a $(K \times K)$ matrix of Markov transition probabilities. The Markov matrix $A$ has a dominant root equal to 1 . Under time-invariant transition probabilities, when this dominant root is unique, the dynamic system (5b) has a unique stationary equilibrium given by $p^{e}=\lim _{t \rightarrow \infty} p_{t}$ for all initial conditions $p_{0}$. This provides a basis to evaluate the long run distribution of $y$. This long run distribution will depend on the underlying dynamics. Again, the long run distribution of $y$ can exhibit many patterns. For example, the long run probability density of $y_{t}$ could exhibit a single peak with little skewness (e.g., under Gaussian shocks and a linear AR process). Alternatively, it could be skewed when the dynamics implies an escape from low outcomes (under resilience) or from high outcomes (under collapse). Finally, it could exhibits multiple peaks (e.g., when a system tends to escape from a zone of instability toward surrounding zones of stability, leading to a bimodal density in the long run). Again, these examples indicate that many patterns of long run distribution are possible, stressing the importance of a flexible approach in the empirical investigation of dynamics.

\section{Econometric Analysis}

Consider the case where equation (3) takes the general form $y_{t}=f\left(y_{t-1}, \ldots, y_{t-p}, x_{t}, e_{t}\right)$ where $x_{t}$ is a vector of explanatory variables affecting $y_{t}$ at time $t$. Define the conditional distribution function of $y_{t}$ as $F\left(v \mid y_{t-1}, \ldots, y_{t-m}, x_{t}\right)=\operatorname{Prob}\left[y_{t} \leq v \mid y_{t-1}, \ldots, y_{t-m}, x_{t}\right]=$ $\operatorname{Prob}\left[f\left(y_{t-1}, \ldots, y_{t-m}, x_{t}, e_{t}\right) \leq v\right]$. The distribution function $F\left(v \mid y_{t-1}, \ldots, y_{t-m}, x_{t}\right)$ is conditional on lagged values $\left(y_{t-1}, \ldots, y_{t-m}\right)$ and on $x_{t}$. Define the associated conditional quantile function as the inverse function $q\left(r \mid y_{t-1}, \ldots, y_{t-m}, x_{t}\right) \equiv$ $\inf _{v}\left\{v: F\left(v \mid y_{t-1}, \ldots, y_{t-m}, x_{t}\right) \geq r\right\}$ where $r \in(0,1)$ is the $r^{t h}$ quantile. When $r=0.5$, this 
includes as special case the conditional median $q\left(0.5 \mid y_{t-1}, \ldots, y_{t-m}, x_{t}\right)$. Both the distribution function $F\left(v \mid y_{t-1}, \ldots, y_{t-m}, x_{t}\right)$ and the quantile function $q\left(r \mid y_{t-1}, \ldots, y_{t-m}, x_{t}\right)$ are generic: they provide a general characterization of the dynamics of $y$. In the rest of the paper, we will make extensive use of the quantile function $q\left(r \mid y_{t-1}, \ldots y_{t-m}, x_{t}\right)$ in the analysis of the dynamics of $y_{t}$.

Relying on the conditional quantile function $q\left(r \mid y_{t-1}, \ldots, y_{t-m}, x_{t}\right)$, we focus our attention on the case where the conditional quantile function takes the form $q\left(r \mid y_{1}, \ldots, y_{t-m}, x_{t}\right)=X\left(y_{t-1}, \ldots, y_{t-m}, x_{t}\right) \beta(r), r \in(0,1)$, where $X(\cdot)$ is a $(1 \times K)$ vector and $\beta(r) \in \mathbb{R}^{K}$ is a $(K \times 1)$ vector of parameters. This restricts the analysis to situations where conditional quantiles are linear in the parameters $\beta(r)$. This specification allows the parameters $\beta(r)$ to vary across quantiles, thus providing a flexible representation of the underlying distribution function and its dynamics. In addition, the function $X\left(y_{t-1}, \ldots, y_{t-m}, x_{t}\right)$ can possibly be nonlinear in $\left(y_{t-1}, \ldots, y_{t-m}\right)$ thus allowing for nonlinear dynamics.

In the analysis presented below, we consider an econometric model specification of the form

$$
q\left(r \mid y_{1}, \ldots, y_{t-m}, x_{t}\right)=\beta_{0}\left(r, x_{t}\right)+\sum_{j=1}^{m} \beta_{j}\left(r, x_{t}\right) y_{t-j} .
$$

To illustrate the flexibility of this specification, note that it reduces to a standard autoregressive model of order $m, \mathrm{AR}(\mathrm{m})$ (e.g., see Enders, 2010), when $\beta_{j}\left(r, x_{t}\right)=\beta_{j}, j=$ $1, \ldots, m$, for all $r \in(0,1)$ and all $x_{t}$, i.e. when the autoregression parameters $\beta_{j}$ 's are constant and do not vary across quantiles. When the intercept $\beta_{0}\left(r, x_{t}\right)$ varies across quantiles $r$, this provide a flexible representation of the distribution function (e.g., it allows for any variance, skewness and kurtosis). Also, when $\beta_{0}\left(r, x_{t}\right)$ varies with $x_{t}$, this allows $x_{t}$ to shift the intercept. But an $\operatorname{AR}(m)$ model is restrictive in two important ways: $1 /$ it is restricted to linear dynamics in 
the mean; and 2/ it does not provide a flexible representation of dynamics in variance, skewness or kurtosis. Such limitations have stimulated more general specifications capturing dynamics in variance (e.g., the generalized autoregressive conditional heteroscedastic (GARCH) model proposed by Bollerslev (1986)) and non-linear dynamics (e.g., Markov switching models (Hamilton, 1989), threshold autoregression (TAR) models (Tong, 1990) and smooth transition autoregressive (STAR) models (Van Dijk et al., 2002)).

When $\beta_{j}\left(r, x_{t}\right)=\beta_{j}(r), j=1, \ldots, m$, the above specification reduces to the Quantile Autoregressive model QAR(m) proposed by Koenker and Xiao (2006). Unlike an AR(m), the QAR $(\mathrm{m})$ model allows the autoregression parameters $\beta_{j}(r)$ to vary across quantiles $r \in(0,1)$, thus permitting dynamics to differ in different parts of the distribution. In the more general case, $\beta_{j}\left(r, x_{t}\right)$ can vary with the explanatory variables $x_{t}$, allowing economic conditions to affect dynamics.

In addition, considering the case where the state space $\mathbb{R}$ is partitioned into $K$ subsets $\mathbb{R}=\left\{S_{1}, \ldots, S_{K}\right\}$, define $d_{k, t-j}=\left\{\begin{array}{l}1 \\ 0\end{array}\right\}$ when $y_{t-j}\left\{\begin{array}{l}\in S_{k} \\ \notin S_{k}\end{array}\right\}, k=1, \ldots, K, j=1, \ldots, m$. Depending on the value taken by the lagged variable $y_{t-j}$, this identifies $K$ regimes $\left(S_{1}, \ldots, S_{K}\right)$ with the $d_{k, t-j}$ 's being variables capturing the switching between regimes, $j=1, \ldots, m$. When $x_{t}$ includes the variables $d_{k, t-j}$ 's, this allows the autoregression parameter $\beta_{j}\left(r, x_{t}\right)$ to vary across the $K$ regimes, $j=1, \ldots, m$. The general case corresponds to a Threshold Quantile Autoregressive (TQAR $(\mathrm{m}))$ model where, for each lag $j, \beta_{j}\left(r, x_{t}\right)$ can vary both across quantiles $r \in(0,1)$ and across regimes (Galvao et al., 2011; Chavas and Di Falco, 2016). When $\beta_{j}\left(r, x_{t}\right)=\beta_{j}\left(x_{t}\right), j=$ $1, \ldots, m$, (i.e., when the autoregression parameters do not vary across quantiles), a TQAR(m) reduces to a threshold autoregression (TAR(m)) model (see Tong (1990)). And as noted above, a 
TQAR $(m)$ model includes as special cases a QAR $(m)$ model (obtained when $\beta_{j}\left(r, x_{t}\right)=$ $\left.\beta_{j}(r), j=1, \ldots m\right)$, as well as a $\mathrm{AR}(\mathrm{m})$ model (obtained when $\left.\beta_{j}\left(r, x_{t}\right)=\beta_{j}, j=1, \ldots m\right)$. In general, a TQAR $(\mathrm{m})$ model is very flexible at representing nonlinear dynamics. Indeed, in a TQAR $(m)$ model and for each lag $j$, the autoregression parameter $\beta_{j}\left(r, x_{t}\right)$ can vary with the value of the current variable $y_{t}$ (as captured by the quantile $r$ ), with the value of the lagged variable $y_{t-j}$ (as captured by the regime-switching variables $d_{k, t-j}$ 's), and with the value of other variables in $x_{t}$. A TQAR $(\mathrm{m})$ model will be used below in our empirical investigation of dynamics.

Consider a sample of $n$ observations on $(y, X)$, where $X$ is a vector of explanatory variables and $q(r \mid X)=X \beta(r), r \in(0,1)$. Denote the $i^{t h}$ observation by $\left(y_{i}, X_{i}\right), i \in N \equiv$ $\{1, \ldots, n\}$. For a given quantile $r \in(0,1)$ and following Koenker (2005), the quantile regression estimate of $\beta(r)$ is

$$
\hat{\beta}(r) \in \operatorname{argmin}_{\beta}\left\{\sum_{i \in N} \rho_{r}\left(y_{i}-X_{i} \beta\right)\right\},
$$

where $\rho_{r}(w)=w[r-I(w<0)]$ and $I(\cdot)$ is the indicator function. As discussed in Koenker (2005), the quantile estimator $\hat{\beta}(r)$ in (7) is a minimum distance estimator with desirable statistical properties. The quantile estimator (7) applied to the dynamic specification (6) will provide the basis for our empirical analysis presented next.

\section{An Application to Wheat Productivity}

Our investigation proceeds studying the dynamics of wheat productivity in Kansas. The analysis involves annual wheat yield in Kansas over the period 1885-2012 (USDA, 2015). This covers the period of the American Dust Bowl (in the 1930's) when the US Great Plains were affected by a major environmental catastrophe. The Dust Bowl was the joint product of adverse 
weather shocks (a major drought) and poor agricultural management. The Dust Bowl is remembered by two of its main features: $1 /$ severe drought leading to crop failure and triggering massive migration out of the western Great Plains; and 2/ soil and wind erosion (Hornbeck, 2012). The Dust Bowl had short term effects on agricultural production (as drought generated crop failure). But it also had longer effects: soil erosion had lasting adverse effects on land productivity (Hornbeck, 2012). Kansas has been the leading wheat producing state in the US (USDA, 2015). As noted in the introduction, this makes studying wheat yield dynamics in Kansas a great case study of the response of productivity to environmental shocks.

The data on wheat yield (t/ha) in Kansas over the period 1885-2012 were obtained from USDA (2015). They are presented in Figure 2. Figure 2 shows three interesting features. First, as expected, the early 1930's (corresponding to the Dust Bowl) is a period exhibiting low yields. Second, wheat yields have been trending upward, especially after 1940, indicating the presence of significant productivity growth and technological progress over the last 70 years. Third, there is much variability in yield over time, reflecting the impact of various environmental shocks (including weather shocks).

Our investigation explores the distribution of wheat yield as if we were farmers. Since weather shocks are mostly unpredictable, it means that the distribution of yield is evaluated ex ante at the beginning of the growing season, i.e. before weather shocks become observable. In the ex-ante assessment, the yield distribution is thus unconditional with respect to all unobservable factors affecting farm productivity (including weather effects). In this context, the investigation of dynamic adjustments in Kansas wheat yield is presented next. 


\section{Preliminary Analysis}

We start with a simple analysis of yield dynamics. With wheat yield $y_{t}$ as the dependent variable, we first estimate simple autoregressive models. Table 1 presents the estimation results for alternative model specifications. Two time trend variables are included in all models: a general time trend $t=y e a r-2000$ and a time trend $t_{1}=\left\{\begin{array}{c}0 \\ y e a r-1935\end{array}\right\}$ when year $=$ $\left\{\begin{array}{l}<1935 \\ \geq 1935\end{array}\right\}$, where $t_{1}$ captures technological progress after 1935. The models include autoregressive models of order $\mathrm{m}, \mathrm{AR}(\mathrm{m})$, with $\mathrm{m}=1,2$. The $\mathrm{AR}(1)$ model shows that lag-1 coefficient is 0.703 and highly significant. This documents the presence of dynamics in yield adjustments. The lagged- 2 coefficient in the AR(2) model is not statistically significant. A formal Wald test of the AR(1) model as a null hypothesis against the AR(2) model gave a pvalue of 0.709 , indicating that there is no significant dynamics going beyond one-period lag.

Table 1 also reports threshold autoregressive models (TAR(m) allowing the autoregression parameters to vary across three regimes $\left(d_{i}, d_{2}, d_{3}\right)$. The regimes are defined such that $d_{i, t}=\left\{\begin{array}{l}1 \\ 0\end{array}\right\}$ when $y_{t}\left\{\begin{array}{l}\in S_{i, t} \\ \notin S_{i, t}\end{array}\right\}, i=1,2,3$, with $S_{1, t}=\left[-\infty, b_{1, t}\right], S_{2, t}=\left(b_{1, t}, b_{3, t}\right]$ and $S_{3, t}=$ $\left(b_{3, t}, \infty\right], b_{1, t}$ and $b_{3, t}$ being respectively the $1 / 3$ and $2 / 3$ quantile of the yield distribution obtained from the AR(1) model reported in Table 1 . Thus, regime 1 means that yield is in the $1 / 3$ lower quantile of the yield distribution; and regime 3 means that yield is in the $1 / 3$ upper quantile of the yield distribution. In this context, having $d_{1, t-1}=1$ corresponds to situations of low lag-1 yield where $y_{t-1}$ is in regime 1 . And having $d_{3, t-1}=1$ corresponds to situations of high lag-1 yield where $y_{t-1}$ is in regime 3 . In TAR(m) models, the autoregression parameters are allowed to shift across the three regimes. For a TAR(1), Table 1 shows that the lag-1 coefficient is 0.409 in regime 2, 0.507 in regime 1 and 0.407 in regime 3. Importantly, the difference in coefficients 
between regime 1 and regime $2(0.098)$ is statistically significant at the 10 percent level. This provides statistical evidence that yield dynamics differ across regimes. This is our first hint of nonlinear dynamics. We also estimated a TAR(2) model. As reported in table 1, the lag-2 coefficients of the TAR(2) model are not statistically significant. A formal Wald test of the TAR(1) model as null hypothesis against a TAR(2) model gave a p-value is 0.959 . Again, this indicates no significant dynamics going beyond one-period lag. On that basis, we continue our analysis based on autoregressive models of order 1 .

Note that all estimated models reported in Table 1 shows that the overall time trend $t$ is not statistically significant, but the effect of the post-1935 time trend $t_{1}$ is always positive and statistically significant. This reflects the presence of significant improvements in agricultural technology over the last 70 years. ${ }^{3}$ Interestingly, the coefficient of the $t_{1}$ variable is smaller in the TAR(1) model (0.017) compared to the AR(1) model (0.023). This indicates that productivity growth interacts with changing dynamics across regimes.

\section{Quantile Dynamics}

Our preliminary analysis found statistical support for a TAR(1) specification. A discussed in section 3, while a TAR model allows the autoregression parameters to vary across regimes, it does not allow them to vary across quantiles of the current yield distribution. We now extend the analysis by considering a threshold quantile autoregression (TQAR) model. As noted, a TQAR model provides a flexible representation of nonlinear dynamics by allowing autoregression parameters to change both across regimes and across quantiles. This section focuses on an ax ante analysis of quantile dynamics. An ex post quantile analysis (conditional on weather shocks) is presented in the next section. 
Table 2 reports parameter estimates of a TQAR(1) model applied wheat yield for selected quantiles $(0.1,0.3,0.5,0.7,0.9)$. The variables are the same as in the TAR $(1)$ model reported in Table 1. Table 2 shows how the dynamics vary across quantiles. We tested the null hypothesis that the regression parameters are the same across quantiles $(0.1,0.5,0.9)$. With 10 degrees of freedom, the chi-square test value was 5.703 with a p-value less than 0.01 . This implies a strong rejection of the TAR(1) model in favor of the TQAR(1) model. Thus, we find statistical evidence that the regression parameters vary across quantiles. Table 2 shows that the lag- 1 coefficient under regime 2 is 0.687 at the 0.1 quantile. This coefficient is larger than for higher quantiles, indicating the presence of stronger dynamics in the lower tail of the yield distribution. Table 2 also reports that, for the 0.1 quantile, the lag- 1 coefficient differs between regime 1 (where $d_{1, t-1}=1$ ) and regime 2 . The difference is 0.234 . Using bootstrapping for hypothesis testing, we find this difference to be statistically significant at the 1 percent level. This provides evidence against a QAR model and in favor of a TQAR specification. The lag-1 coefficient for the 0.1 quantile is 0.921 under regime 1 (when lagged yield is low), which is much higher than under the other regimes. This documents the presence of much stronger dynamics in the lower tail of yield distribution and when lagged yield is low. This is one of our key findings: dynamic yield adjustments to shocks become quantitatively very different under repeated adverse shocks. As we show below, this is a scenario where adjustments also become qualitatively different.

In addition, Table 2 shows the effects of the $t_{1}$ trend variable are much stronger in the upper tail of the distribution. This indicates that technological progress has contributed to a rapid increase in the upper tail of the yield distribution. But such effects are weaker in the lower tail of the distribution. This reflects significant shifts in the shape of the yield distribution over time (as further discussed below). 
To conduct robustness checks, we explored issues related to the number of lags used in our dynamic analysis. While Table 2 reports estimates for an TQAR(1) model, we also estimated a TQAR(2) model. In a way consistent with the results shown in Table 1, we found that none of the lag-2 coefficients were statistically significant. This indicates that the TQAR(1) model provides an appropriate representation of dynamics. On that basis, the analysis presented in the rest of the paper focuses on a model with one-period lag.

\section{Implications}

Our estimated TQAR(1) model provides a refined representation of the nonlinear dynamics of yield. As noted in section 3, it allows for flexible patterns of stability and instability. To explore in more details the nature and implications of these patterns, we estimate our TQAR(1) model for all quantiles, thus providing a representation of the whole distribution of wheat yield and its dynamics.

First, we use our TQAR(1) model estimated for all quantile to evaluate the distribution function of wheat yield at selected sample points. The resulting simulated distribution is presented in Figure 3 for selected years $(1950,1970,1990,2010)$. As expected, over time, the distribution shifts strongly to the right, reflecting the major effects of technological progress on agricultural productivity. Interestingly, the yield distribution exhibits greater spread (and thus greater risk exposure) in 2010 than in previous years, indicating an increase in the magnitude of unpredictable shocks (possibly due to climate change).

Next, using equation (4), we examine the dynamic properties of our estimated TQAR(1)

by evaluating the associated root $\lambda=\frac{\partial H}{\partial y_{t-1}}$. Under nonlinear dynamics, this root varies with the situation considered. As discussed in section 2, dynamics is locally stable (unstable) at points 
where $\lambda<1(>1)$. We calculated the root $\lambda$ for all quantiles and all three regimes. The results are reported in Figure 4. Figure 4 document the patterns of nonlinear dynamics associated with our estimated TQAR(1) model. It shows three important results. First, from Figure 4, the root $\lambda$ is similar across all three regimes for quantiles greater than 0.3 ; but it exhibits different dynamics for lower quantiles (less than 0.3). More specifically, compared to other regimes, the root $\lambda$ is larger under regime 1 (when lagged yield is low) and in the lower tail of the distribution. This is consistent with the discussion of Table 2 presented in the previous section.

Second, Figure 4 shows that the root $\lambda$ remains in the unit circle (with $|\lambda|<1$ ) in many situations, including regimes 2 and 3 (when lagged yield are not low) or the absence of adverse current shock (for quantiles greater than 0.2). This implies that the system is locally stable in many situations, especially in situations excluding adverse shocks. This is an important result: investigating dynamics in situations around or above the median could only uncover evidence of local stability. As discussed in section 2, this would preclude finding any evidence of traps.

Third, Figure 4 shows that the root $\lambda$ can be larger than 1 but only in situations of successive adverse shocks, i.e. when both $y_{t}$ and $y_{t-1}$ are in the lower tail of the yield distribution. Associating $\lambda>1$ with local instability, we thus find evidence of local instability in the presence of adverse shocks. This has several implications. First, we have identified a zone of local dynamic instability, i.e. a zone of tipping points where the system tends to escape from. Second, associating a zone of instability with successive adverse shocks is an important finding. This raises the question: Is the zone of instability associated with resilience? Or is it associated with a trap or collapse? It depends on the path of escape. As discussed in section 2, if the escape from the zone of instability is toward more favorable situations, the system would be characterized as resilient (e.g., as represented by Scenario 3 in Figure 1). Alternatively, if the 
escape is toward more unfavorable situations, the system may be experiencing a trap or a collapse (e.g., Scenario 4 in Figure 1).

In our case, the zone of instability occurs only under successive unfavorable shocks generating very low yields. It suggests that starting in this zone, there is only one place to go: toward higher yields. This suggests that our zone of instability may be associated with a resilient system that tends to escape from low productivity toward higher productivity under adverse shocks. Indeed, Figure 4 exhibits patterns that are similar to Scenario 3 in Figure 1. To examine this issue in more details, we consider the Markov chain representation of our TQAR(1) model, as given in equations (5a)-(5b). Using $K=50$ and evaluated under conditions occurring in 1995, we obtained the Markov matrix $A$ in (5b). The matrix $A$ has a unique unit root, indicating that the Markov chain is stationary and has a long run distribution. The second root of $A$ has modulus 0.37, indicating a fairly fast adjustment toward the long run distribution. The evolution of the probability function of wheat yield was simulated from equation (5b), starting from a uniform distribution over the range of the data. Starting at $t=0$, the simulated probabilities are reported in Figure 5 under three scenarios: in the short run (after 2 periods, $t=2$ ), in the intermediate run (after 4 periods, $t=4$ ) and in the long run (after 200 periods, $t=200$ ). Figure 5 shows several important results. First, the adjustments toward the steady-state probability function occurs fairly quickly. Second, the simulated probability functions depart from the normal distribution in two ways: $1 /$ they exhibit multiple peaks; and 2/ they are skewed, with a left tail that is much longer than the right tail. Indeed, in all scenarios, Shapiro-Wilk tests of normality have a p-value less than 0.01 , providing strong evidence of departure from normality. Third, Figure 5 shows that the probability of being in the left tail of the probability function declines fast as one moves forward in time (as t goes from 2 to 4 to 200). This implies a dynamic escape from unfavorable events 
located in the lower tail of the distribution. Since escaping an unfavorable zone is the essence of resilience, if follows that Figure 5 documents the presence of resilience. In other words, our estimated TQAR(1) model applied to wheat yield dynamics has two key characteristics: 1/ a zone of instability occurs in the presence of successive unfavorable shocks; and 2/ resilience arises as the underlying dynamic process tends to escape from this unfavorable zone. The significance of these findings is further discussed in section 8 below.

As discussed in section 2, the TQAR model reported in Table 2 is a reduced form model. While a reduced form model provides a valid representation of dynamics, it does not provide structural information on the nature of dynamics. In the Kansas agro-ecosystem, a major source of shocks comes from the weather. Indeed, the Dust Bowl was the result of a major drought that hit the Western Great Plains in the 1930's. This suggests evaluating a structural model where weather variables are explicit determinants of Kansas wheat yield. On that basis, we also specified and estimated a dynamic model of wheat yield including the effects of three weather variables: rainfall in the previous fall rainf, rainfall in the spring rains and average temperature during the growing season temp. Data on these variables were obtained from Burnette et al. (2010), Burnette and Stahle (2013) and NOAA (2016). ${ }^{4}$ These weather variables were introduced in the model both as intercept shifters and as interactions with lagged yield. These interaction effects allow yield dynamics to vary with weather conditions. Estimates of the associated quantile regression equation is presented in Table A1 in the Appendix. As expected, Table A1 shows that weather has statistically significant effects on yield. Rainfall in the previous fall has a positive effect on yield, especially on the lower tail of the distribution. Temperature has a negative effect on yield through its interaction effect with lagged yield, especially in the upper tail of the distribution. This documents that both drought and high temperature have adverse 
effects on agricultural productivity. Such results are consistent with previous research (e.g., Tack et al., 2012, 2015). Table A1 also shows the presence of dynamics. Lagged yield has statistically significant effects on current yield either directly or through its interaction with temperature.

To evaluate the nature of dynamics in the structural model reported in table A1, we calculated the root of the estimated dynamic process across quantiles. Interestingly, we found that the root varies between -0.2 and +0.6 depending on the evaluation point. The root is always in the unit circle for any quantile or any weather condition within the range of data. This implies global stability. Thus, the dynamic model reported in Table A1 does not show any evidence of instability. This contrasts with the reduced form model reported in table 2 (which exhibits local instability as discussed above). ${ }^{5}$ While this result is somewhat surprising, it has two important implications. First, since controlling for weather effects implies the disappearance of instability, it means that there is close association between instability and weather shocks. In other words, our reduced-from evidence of instability must be linked with weather shocks. Second, weather being mostly unpredictable, we do not expect much dynamics in the determination of weather shocks. This indicates that any linkage between weather and yield dynamics must be because of the dynamic response of management and policy to weather shocks. We expand on this interpretation below.

\section{Discussion}

From our reduced form model, our first finding is about local dynamic instability arising but only under unfavorable shocks. This is important. It suggests that a search for local instability is unlikely to be successful if the analysis focuses on "average conditions". This can be problematic in economic research to the extent that most econometric analyses involve 
estimating means or conditional means. While studying the properties of means and conditional means can be interesting, it neglects key information related to events located in the tails of the distribution. In a stochastic context, our findings indicate a need to expand analyses with a focus on dynamics associated with rare and unfavorable events. This is a very intuitive argument. On the positive side, increasing resilience is about improving the odds of escaping the long term effects of facing adverse shocks. On the negative side, avoiding collapse or traps is about reducing the odds of facing adverse conditions and increasing the odds of escaping toward better outcomes. All escape scenarios are about identifying local instability. Our TQAR model provides a good basis to support such inquiries.

Our second finding is also very interesting: applied to wheat yield dynamics, our analysis uncovered evidence of resilience as local instability tends to create an escape away from unfavorable events toward improved outcomes. But it also raises questions about the process supporting such dynamics. Below, we reflect on this process and the interpretations and implications of our findings.

As noted above, our analysis has relied on an ex ante analysis of yield dynamics and focused on assessing the distribution of yield based on information available at the beginning of each growing season. Since weather conditions are mostly unpredictable, we treated the effects of rainfall and temperature during the growing season as part of the shocks represented by the yield distribution function. This raises the question: what constitutes an adverse shock? Much research has examined the determinants of wheat yield (e.g., Olmstead and Rhode, 2011; Tack et al., 2015). Both rainfall and temperature are major factors affecting wheat yield (e.g., Tack et al., 2015; Chavas and Di Falco, 2016). In particular, farming in the western Great Plains faces much rainfall uncertainty as it has experienced repeated periods of severe droughts (Burnette and 
Stahle, 2013). One of the most severe drought occurred in the 1930's: it led massive crop failures and to the Dust Bowl. Because of the massive soil erosion it generated, the Dust Bowl is often seen as an environmental catastrophe (Hornbeck, 2012). Yet, our evidence of resilience suggests a different interpretation.

First, the Dust Bowl induced significant changes in agricultural management and policy. A major federal policy change was the creation of the Soil Conservation Service (SCS) in 1935. The SCS played a major role of reducing the incidence of wind erosion in the Western Great Plains (Hurt, 1981). The circumstances under which the SCS was created are of interest. Starting in 1932, severe droughts caused widespread crop failure in the Great Plains, exposing the soil to blowing winds and generating large dust storms. On March 61935 and again on March 21 1935, dust clouds passed over Washington D.C. and darkened the sky as Congress was having hearings on soil conservation legislation. This motivated policy maker to act: The Soil Conservation Act was signed by President Roosevelt on April 27, 1935, creating the Soil Conservation Service (SCS) in USDA. This was an example of rather fast policy response to a crisis.

The Dust Bowl also stimulated significant adjustments in agricultural management. The SCS established demonstration projects to persuade farmers to adopt more sustainable tillage and cropping practices (including contour plowing, terracing, strip cropping, planting drought resistant crops and greater reliance on pasture). For participating farmers, the SCS programs contributed to improving farm practices, increasing land values and boosting farm income (Hurt, 1981). As a result, farmers shifted land from wheat into hay and pasture; and they implemented new soil conservation techniques (Hornbeck, 2012, p. 1480). Such changes helped mitigate the adverse effects of severe droughts. 
Second, the Dust Bowl did not start a process of desertification of the Western Great Plains. On the contrary, cultivated farmland increased during the 1930's and 1940's (Hornbeck, 2012, 1480-1490). This indicates that the 1930's droughts stimulated major innovations in agricultural management and policy. To the extent that these changes reduced the adverse effects of droughts, they contributed to creating a more resilient agro-ecological system.

Thus, we associate our evidence of resilience with induced innovations in both policy and management that followed the Dust Bowl. This interpretation raises the question: What would have been the effects of the Dust Bowl without such innovations? Of course, this is a hypothetical scenario that we have not observed. Still, we can hypothesize about what might have happened. First, our evidence of resilience would likely disappear. For example, without innovations, continued soil erosion may have led to the desertification of the Western Great Plains. Under this scenario, the adverse long term effects of the Dust Bowl assessed by Hornbeck (2012) would have been much worse. The agro-ecosystem of the Western Great Plains may have collapsed. In this case, the zone of instability identified in Figure 4 would move to the right. In the context of Figure 1, this would correspond to a move from scenario 3 (resilience) toward scenario 2 or even toward scenario 4 (collapse). The process of collapse would occur when adverse shocks put the system in the zone of instability with a tendency to move toward lower outcomes (e.g., scenario 4 in Figure 1). Figure 5 would also change. Under collapse, the lower tail of the yield distribution would become much thicker. And the probability function may exhibit multiple peaks in the lower tail, with a new peak possibly rising in the extreme lower tail (corresponding to collapse). In this case, a key issue would be whether "valleys" exist inbetween peaks in the probability density function. The presence of valleys would indicate that there are positive probabilities of escaping the lower tail of the distribution. Alternatively, the 
absence of such valleys would mean any collapse obtained under adverse shocks would be irreversible.

Of course, these hypothetical scenarios differ from the ones reported in Figure 4 and 5. Yet, our discussion has three important implications. First, evaluating resilience/collapse/traps must focus on the nature of dynamics under adverse shocks. As noted above, just knowing what is happening "on average" is not sufficient. Second, the assessment of local instability is crucial. Our TQAR approach provides a great analytical framework to conduct this assessment. Third, in general, the dynamic response to adverse shocks depends on management and policy. Our discussion has pointed out the role of innovations. On the negative side, collapse/traps are more likely to arise in the absence of management and policy response to adverse shocks. On the positive side, induced innovations in management and policy can be a crucial part of designing a more resilient system. Our analysis indicates the important role played by the induced response of management and policy to adverse shocks.

\section{Conclusion}

This paper has studied the dynamic response to shocks, with an application an agroecosystem productivity. It has proposed a threshold quantile autoregression (TQAR) model as a flexible representation of stochastic dynamics. It has focused on the identification of zones of local instability and their usefulness in the characterization of resilience and traps. The usefulness of the approach was illustrated in an application to the dynamics of wheat yield in Kansas. The analysis examined the effects of extreme shocks both in the short run and in the long run. It identified a zone of instability in the presence of successive adverse shocks. It also 
finds evidence of resilience. We associate the resilience with induced innovations in management and policy in response to adverse shocks.

Our approach is generic and can be applied to the analysis of dynamics in any economic system. Our empirical analysis focused on a particular agro-ecosystem. Our findings documented the role of local instability in response adverse shocks. Such findings are expected to vary across economic situations. This motivates a need to extend our analysis and its applications to other economic conditions. 


\section{References}

Barrett, C.B. and M.R. Carter. (2013). “The Economics of Poverty traps and Persistent Poverty: Empirical and Policy Implications" Journal of Development Studies 49: 976-990.

Billingsley, P. (1961). Statistical Inference for Markov Processes. University of Chicago Press.

Bollerslev, T. (1986). "Generalized Autoregressive Conditional Heteroscedasticity" Journal of Econometrics 31: 307-327.

Burnette, D.J., D.W. Stahle and C.J. Mock. (2010). "Daily-Mean Temperature Reconstructed for Kansas from Early Instrumental and Modern Observations" Journal of Climate 23: 13081333.

Burnette, D.J. and D.W. Stahle. (2013). "Historical Perspective on the Dust Bowl Drought in the Central Unites States" Climate Change 116: 479-494.

Chavas, J.P. and S. Di Falco. (2016). "Resilience and Dynamic Adjustments in Agroecosystems: The Case of Wheat Yield in England" Environmental and Resource Economics (forthcoming).

Common, M. and C. Perrings. (1992). "Towards an ecological economics of sustainability" Ecological Economics 6: 7-34.

Derissen S., M.F. Quaas and S. Baumgärtner. (2011). “The relationship between resilience and sustainable development of ecological-economic systems" Ecological Economics 70: $1121-1128$.

Diamond, J.M. (2005). Collapse: How Societies Choose to Fail or Succeed. Viking Books, New York.

Di Falco, S. and J.P. Chavas. (2008). "Rainfall Shocks, Resilience and the Effects of Crop Biodiversity on Agroecosystem Productivity" Land Economics 64: 83-96. 
Enders, W. (2010). Applied Econometric Time Series, Third Edition, Wiley, New York.

Folke, C., S.R. Carpenter, B.H. Walker, M. Scheffer, T. Elmqvist, L.H. Gunderson and C.S. Holling. (2004). "Regime shifts, resilience, and biodiversity in ecosystem management". Annual Review of Ecology, Evolution and Systematics 35: 557-581.

Galvao, A., G. Montes-Rojas and J. Olmo. (2011). "Threshold Quantile Autoregressive Model” Journal of Time Series Analysis 32: 253-267.

Gunderson, L.H. (2000). "Ecological resilience in Theory and Application" Annual Review of Ecological Systems 31: 425-439.

Hasselblatt, B. and A. Katok. (2003). A First Course in Dynamics. Cambridge University Press, New York.

Hamilton, J.D. (1989). “A New Approach to the Economic Analysis of Nonstationary Time Series and the Business Cycle” Econometrica 57: 357-384.

Holling, C.S. (1973). "Resilience and Stability of Ecological Systems" Annual Review of Ecology and Systematics 4: 1-23.

Hornbeck, R. (2012). “The Enduring Impact of the American Dust Bowl: Short- and Long-Run Adjustments to Environmental Catastrophe” American Economic Review 102 (1): 14771507.

Hurt, D.R. (1981). The Dust Bowl: An Agricultural and Social History. Nelson-Hall, Chicago.

Koenker, R. (2005). Quantile Regression. Cambridge University Press, Cambridge.

Koenker, R. and Z. Xiao. (2006). "Quantile Autoregression” Journal of the American Statistical Association 101: 980-990.

Kraay, A. and D. McKenzie. (2014). “Do Poverty Traps exists? Assessing the Evidence” Journal of Economic Perspectives 28: 127-148. 
May, R.M. (1976). "Simple mathematical models with very complicated dynamics." Nature 261: 459-467.

Meyn, S.P. and R.L. Tweedie. (1993). Markov Chains and Stochastic Stability. Springer-Verlag, London.

NOAA. (2016). Global Historical Climatology Network (GHCN). National Center for Environmental Information. National Oceanographic and Atmospheric Administration. United States Department of Commerce.

Olmstead, A.L. and P.W. Rhode. (2011). "Adapting North American Wheat Production to Climatic Challenges, 1839-2009" Proceedings of the National Academy of Sciences 108: $480-485$.

Perrings, C. (1998). "Resilience in the Dynamics of Economy-Environment Systems" Environmental and Resource Economics 11: 503-520.

Tack, J., A. Harri and K. Coble. (2012). "More than Mean Effects: Modeling the Effect of Climate on the Higher Order Moments of Crop Yields" American Journal of Agricultural Economics 94: 1037-1054.

Tack, J., A. Barkley and L.L. Nalley. (2015). "Effect of Warming Temperatures on US Wheat Yields" Proceedings of the National Academy of Sciences 112: 6931-6936.

Tainter, J.A. (1990). The Collapse of Complex Societies. Cambridge University Press, Cambridge.

Tong, H. (1990). Non-linear Time Series: A Dynamical System Approach. Clarendon Press, Oxford.

USDA. (2015). “Kansas Wheat History” United States Department of Agriculture, National Agricultural Statistics Service, Northern Plains regional Field Office, Lincoln, NE. 
Van Dijk, D., T. Teräsvirta, and P.H. Franses. (2002). Smooth Transition Autoregressive Models - A Survey of Recent Developments. Econometric Reviews 21: 1-47. 
Table 1: Estimates of Autoregressive Models

\begin{tabular}{|c|c|c|c|c|}
\hline Paramaters & AR(1) & AR(2) & TAR(1) & TAR(2) \\
\hline Intercept & $0.703^{* * *}$ & $0.706^{* * *}$ & $0.543 * * *$ & $0.540^{* *}$ \\
$y_{t-1}$ & $0.236^{* * *}$ & $0.219 * *$ & $0.409 * *$ & $0.463 * *$ \\
$y_{t-2}$ & & 0.034 & & -0.039 \\
$d_{1, t-1} * y_{t-1}$ & & & $0.098^{*}$ & $0.111^{*}$ \\
$d_{3, t-1} * y_{t-1}$ & & & -0.010 & -0.018 \\
$d_{1, t-2} * y_{t-2}$ & & & & -0.020 \\
$d_{3, t-2} y_{t-2}$ & & & -0.002 & -0.011 \\
$t$ & -0.002 & -0.003 & $0.017 * *$ & $0.017 * *$ \\
$t_{1}$ & $.0023^{* * *}$ & $0.024 * * *$ & & 0.860 \\
\hline$R^{2}$ & 0.857 & 0.856 & 0.859 & \\
\hline
\end{tabular}

Note: Asterisks indicate the significance level: * for the 10 percent significance level; *** for the 5 percent significance level; and $* * *$ for the 1 percent significance level.

Table 2: Estimates of Threshold Quantile Autoregressive Model TQAR(1) for Selected Quantiles

\begin{tabular}{|c|c|c|c|c|c|}
\hline \multirow{2}{*}{ Parameters } & \multicolumn{5}{|c|}{ quantile } \\
\cline { 2 - 6 } & $\mathbf{r = 0 . 1}$ & $\mathbf{r}=\mathbf{0 . 3}$ & $\mathbf{r}=\mathbf{0 . 5}$ & $\mathbf{r = 0 . 7}$ & $\mathbf{r}=\mathbf{0 . 9}$ \\
\hline Intercept & -0.003 & $0.481 * *$ & $0.747 * * *$ & $1.036^{* * *} *$ & $1.409^{*} * *$ \\
$y_{t-1}$ & $0.687 * * *$ & 0.407 & 0.272 & -0.036 & -0.341 \\
$d_{1, t-1} * y_{t-1}$ & $0.234 * * *$ & 0.086 & -0.015 & -0.026 & -0.007 \\
$d_{3, t-1} * y_{t-1}$ & -0.067 & -0.057 & -0.051 & 0.125 & 0.156 \\
$t$ & 0.000 & -0.003 & $-0.005 * *$ & $-0.005 * *$ & -0.001 \\
$t_{1}$ & 0.003 & $0.018^{* *}$ & $0.027 * * *$ & $0.035^{* * *}$ & $0.040^{* * *}$ \\
& & & & & \\
\hline
\end{tabular}

Note: Hypothesis testing is conducted using bootstrapping. Asterisks indicate the significance level: * for the 10 percent significance level; *** for the 5 percent significance level; and *** for the 1 percent significance level. 
Figure 1: Dynamic Patterns for the dominant root $\left|\lambda_{1}\right|$

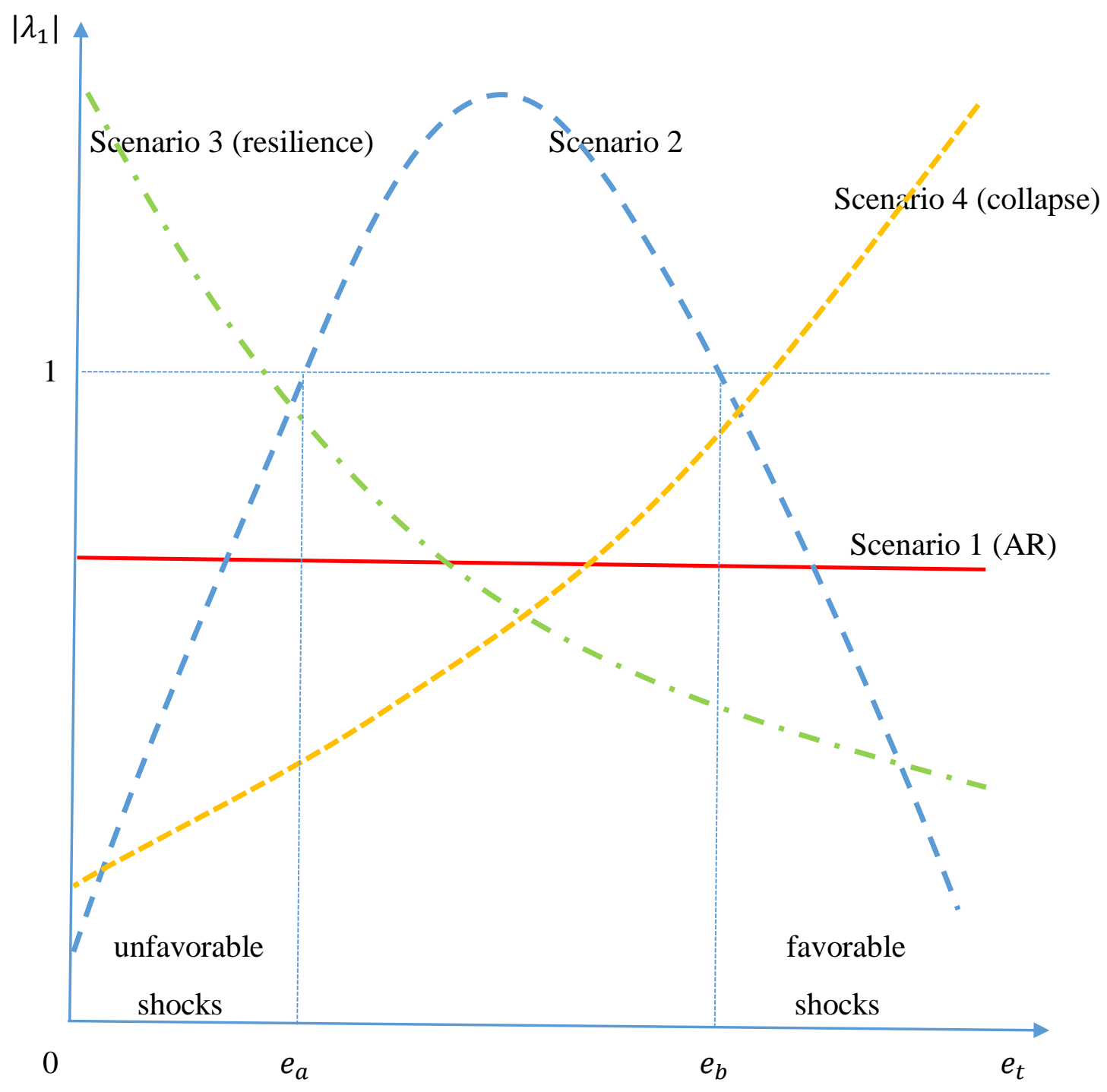


Figure 2: Kansas Wheat Yield (ton per ha)

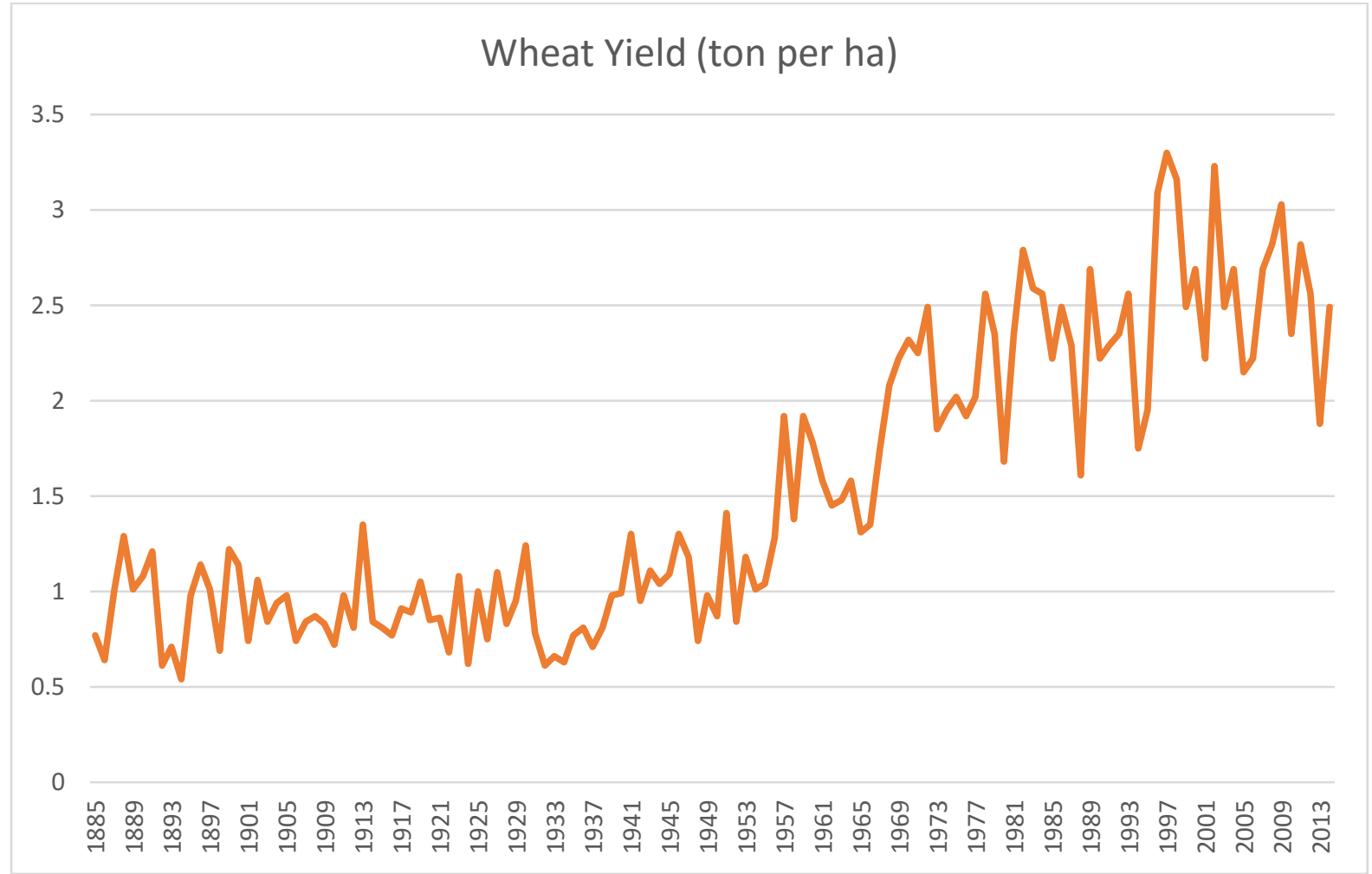


Figure 3: Simulated Yield Distribution

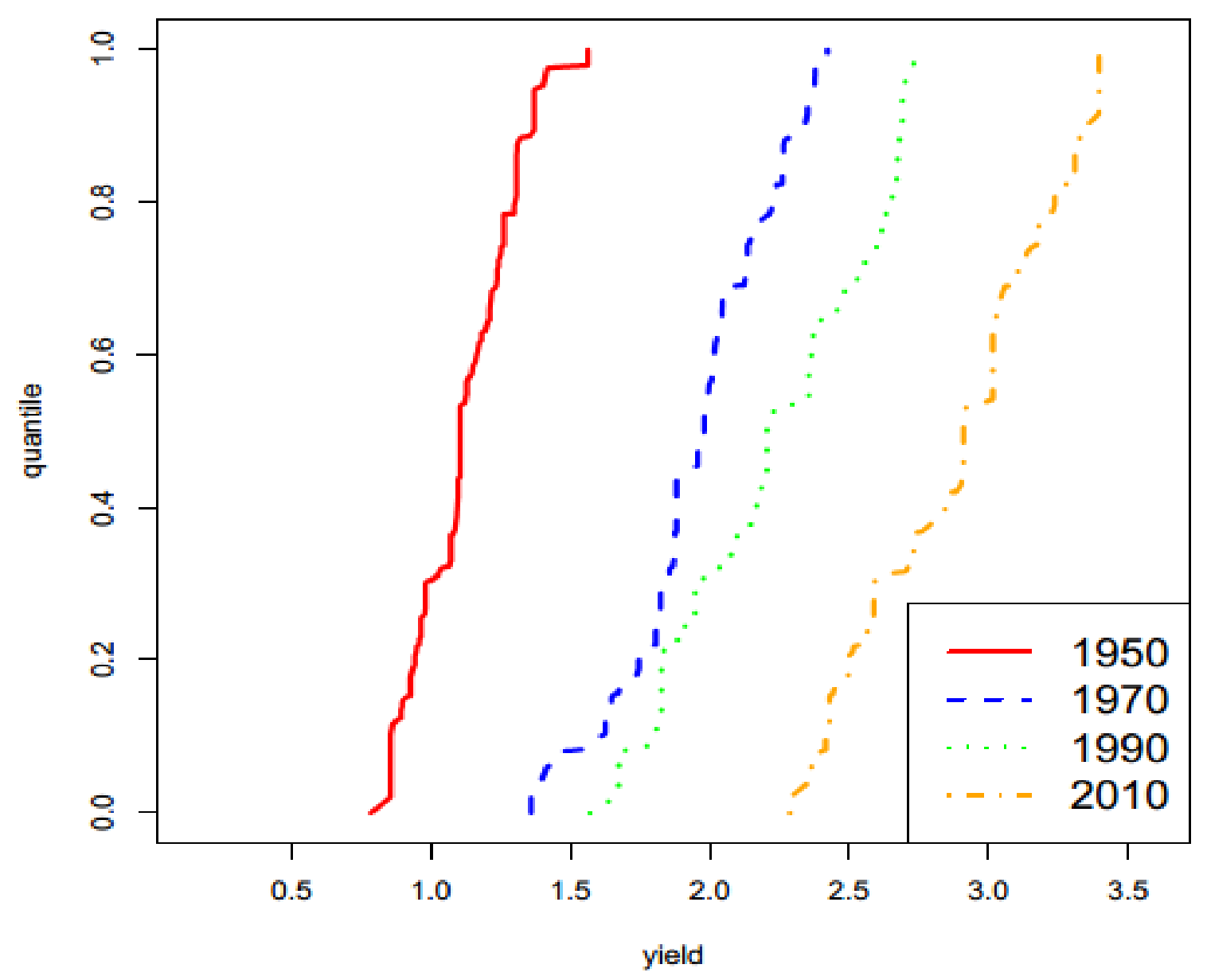


Figure 4: Root of the Dynamic Yield Equation

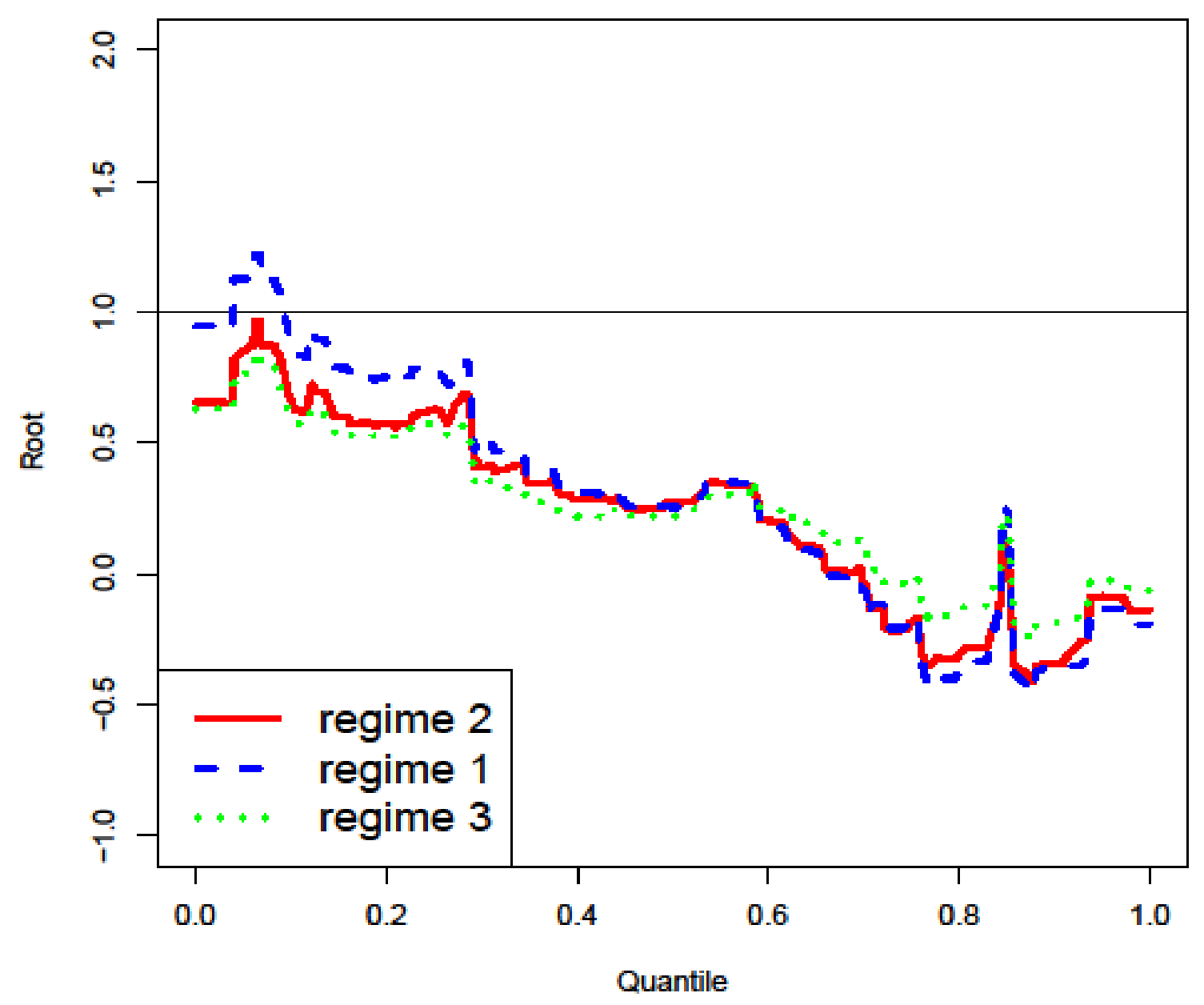


Figure 5: Simulated Probability Function of Wheat Yield in the Short Run $(t=2)$, Intermediate Run $(\mathrm{t}=4)$ and Long Run $(\mathrm{t}=200)$

Simulated Probabilities

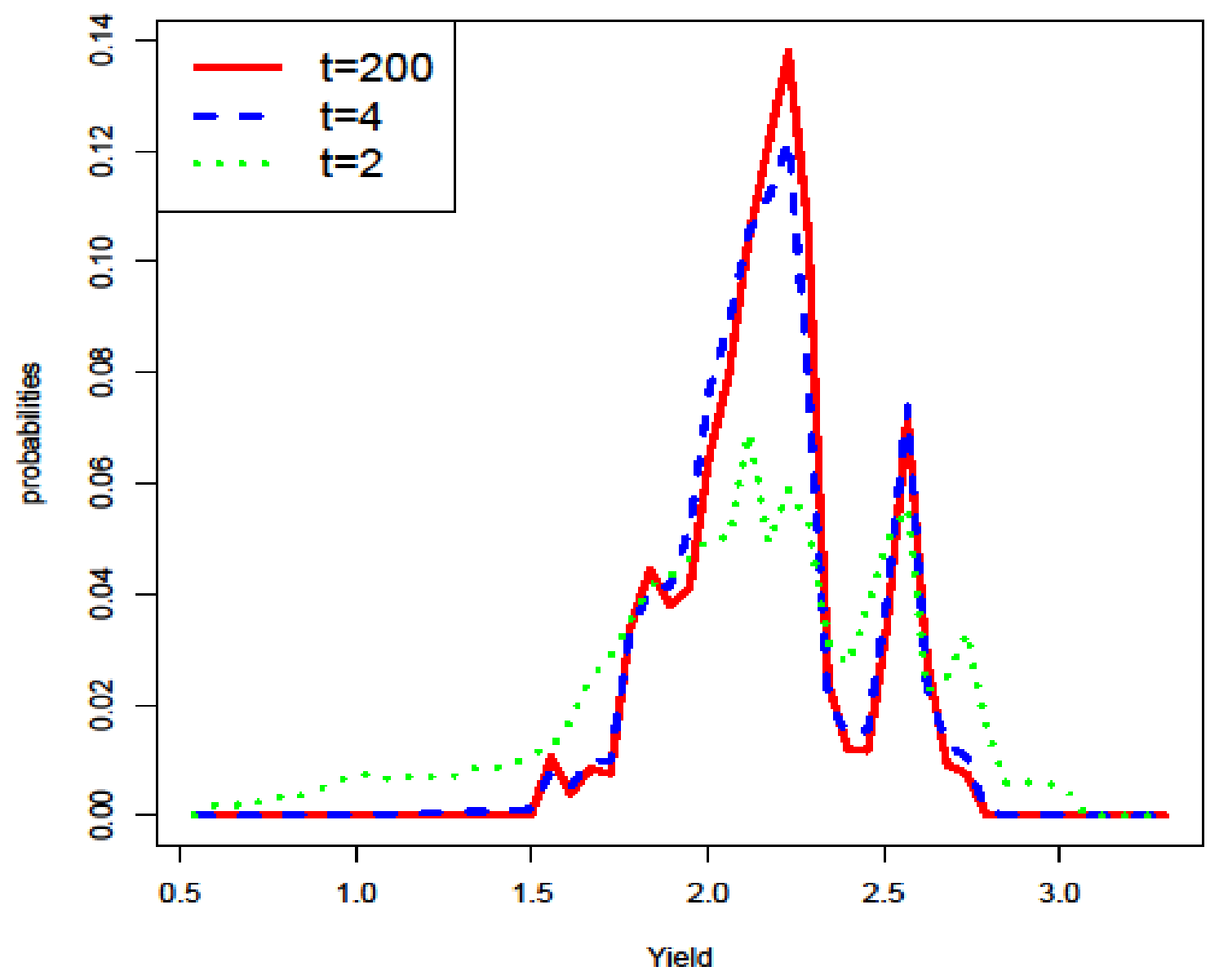




\section{Appendix}

Table A1: Estimates of Quantile Autoregressive Model of Wheat Yield including Weather Shocks, Selected Quantiles

\begin{tabular}{|c|l|l|l|l|l|}
\hline \multirow{2}{*}{ Parameters } & \multicolumn{5}{|c|}{ quantile } \\
\cline { 2 - 6 } & $\mathbf{r = 0 . 1}$ & $\mathbf{r}=\mathbf{0 . 3}$ & \multicolumn{1}{|c|}{$\mathbf{r = 0 . 5}$} & $\mathbf{r}=\mathbf{0 . 7}$ & $\mathbf{r}=\mathbf{0 . 9}$ \\
\hline Intercept & 0.44929 & 0.02457 & 0.11511 & -0.09011 & -0.06440 \\
$y_{t-1}$ & 0.22780 & 0.23496 & $0.29597 * *$ & 0.18766 & $0.19131 * *$ \\
rain_s & -0.00045 & -0.00043 & 0.00055 & 0.00027 & 0.00025 \\
rain_f & $0.00094 *$ & 0.00071 & 0.00089 & $0.00119 * *$ & 0.00074 \\
temp & -0.00081 & 0.00994 & 0.00584 & 0.01346 & 0.01549 \\
rain_s $* y_{t-1}$ & 0.00057 & 0.00060 & -0.00068 & -0.00040 & -0.00036 \\
temp $* y_{t-1}$ & -0.00395 & -0.01454 & -0.01458 & $-0.02326 * * *$ & $-0.02495 * * *$ \\
$t$ & -0.00076 & -0.00108 & -0.00356 & $-0.00520 * *$ & -0.00093 \\
$t_{1}$ & $0.01823 * * *$ & $0.02043 * * *$ & $0.02751 * * *$ & $0.0356 * * * 5$ & $0.03070 * * *$ \\
\hline
\end{tabular}

Note: Hypothesis testing is conducted using bootstrapping. Asterisks indicate the significance level: * for the 10 percent significance level; *** for the 5 percent significance level; and *** for the 1 percent significance level. 


\section{Footnotes}

${ }^{1}$ Note that assuming serial independence of $e_{t}$ is not restrictive since any serial correlation can be captured by the dynamic equation for $z_{t}$ in (1b).

${ }^{2}$ Other possible scenarios (not shown in Figure 1) are when there is a zone of stability surrounded by zones of instability, or when instability is global (e.g., under chaos).

${ }^{3}$ Note that technological progress involves many factors, including improved wheat varieties, increased use of fertilizer, greater reliance on irrigation, and improved farm management practices.

${ }^{4}$ Rainfall is measured in millimeters and temperature in degree Celsius.

${ }^{5}$ Note that Chavas and Di Falco (2016) obtained a similar result for English wheat. 